\title{
INFLUENCE OF NUTRIENTS IN SOIL AND VINE LEAVES AND METEOROLOGICAL FACTORS UPON VINE CROP AND MUST QUALITY
}

\author{
FILIP KRAIC ${ }^{1}$, JAN MOCAK ${ }^{1,2}$, MIROSLAV ARGAY ${ }^{3}$ \\ ${ }^{1}$ Department of Biotechnology, University of SS. Cyril and Methodius, J. Herdu 2, \\ SK-917 01,Trnava, (filip.kraic@ucm.sk) \\ ${ }^{2}$ Institute of Analytical Chemistry, Slovak University of Technology, Radlinskeho 9, \\ SK-812 37 Bratislava \\ ${ }^{3}$ Research Institute of Oenology and Viticulture, Matuskova 25, SK-831 01 Bratislava
}

\begin{abstract}
The aim of this work was to analyze the supply of vine by nutrients by determining the content of nitrogen, phosphorus, potassium, calcium and magnesium in the vineyard soil and the vine leaves. Its further goal was to discover mutual relationships among the content of determined nutrients, the vine crop, and the quality of the produced must. Must quality was here defined by the contents of sugar and acids. The final goal was to investigate the relations among the nutrients, vine crop and its quality and meteorological factors measured during the whole year cycle, and especially at the time of important vegetation changes. The necessary evaluations were performed using multidimensional data analysis.
\end{abstract}

Key words: vine, soil analysis, nutrients, electroultrafiltration, principal component analysis, discriminant analysis

\section{Introduction}

The relations among the nutrients content in vine leaves and in the soil extracts are complicated and established by the concentration levels as well as antagonistic/ synergic interrelations of the individual influences. The applicable agrochemical methods of the soil analysis utilize different extraction agents of different concentration and ionic strength (NÉMETH, 1979; NÉMETH, 1985). The nutrients were extracted by different solvents: water (electroultrafiltration method), calcium lactate solution adjusted to $\mathrm{pH} 3.7$ (phosphorus determination by Egner), strong polar solvents like the mixture of acetic acid, ammonium fluoride and ammonium chloride in hydrochloric acid (Melich method), and the mixture of ammonium acetate and ammonium oxalate (potassium by Schachtschabel). Different analytical methods were used also for the nutrients analysis in leaves, where the concentration of the most important elements was determined. All corresponding results together with meteorological factors (expressed mainly by temperature, quantity of rainfall and extent of the sunshine in individual vegetation cycles) represent a large number of factors related to the vine crop, must quality, and consequently, to the quality of the produced wine.

The primary goal of this work was to determine the supply of vine by nutrients, which was obtained by analyses of nitrogen, phosphorus, potassium, calcium and magnesium in the vineyard soil and the vine leaves. The second goal was to discover 
the mutual relationships among the determined content of nutrients and three target variables: vine crop, sugar content and content of acids. The last goal was to uncover the hidden relations among the nutrients, vine crop and its quality (sugar, acids) and the meteorological factors measured during the whole year cycle, mainly in the time of important vegetation changes.

\section{Material and methods}

\subsection{Analytical determinations}

The extractions of nutrients present in soil were made in two ways: (a) by water (when using electroultrafiltration method), (b) by $0.21 \mathrm{~mol} / \mathrm{l}$ ammonium chloride in $0.012 \mathrm{~mol} / \mathrm{l}$ hydrochloric acid (when Melich method was applied). Determinations of nutrients in leaves were performed after the leaves had been dried and mineralized. For determining the nutrient elements $\mathrm{P}, \mathrm{K}, \mathrm{Ca}$, and $\mathrm{Mg}$ in soil two quantitative methods were used: electroultrafiltration (NÉMETH, 1982; MENGEL and KIRKBY, 2001) and Melich method (MELICH, 1984; JONES, 2001).

\subsection{Exploited data}

The studied data originated from the Research Institute of Oenology and Viticulture in Bratislava, Slovakia. The number of sampling sites, all localized in Southern Slovakia, was 24. The total number of the considered variables (factors) was 41, among them 4 target variables (vine crop, sugar content in must, content of acids in must, and year of the vine production), 10 variables concerning vine leaves - given by the content of 5 elements (N, P, K, Ca, Mg) at the beginning (April - May, denoted by number 1, e.g. K1) and the end (September - October, denoted by 2) of the vegetation cycle, 8 variables concerning the vineyard soil - given by the content of 4 elements ( $\mathrm{P}, \mathrm{K}, \mathrm{Ca}, \mathrm{Mg}$ ) found by two analytical methods (electroultrafiltration and Melich), and 19 meteorological factors - given by 7 temperature indicators T4, T5, T9, $\mathrm{T} 10, \mathrm{~T} 1 \mathrm{t} 12, \mathrm{~T} 4 \mathrm{t} 9, \mathrm{~T} 4 \mathrm{t} 10$ (with the number related to the month or to the time interval between two months), 6 rainfall indicators Rain4, Rain5, Rain9, Rain10, Rain1t12, Rain4t10, and finally 6 sunshine indicators Sun4, Sun5, Sun9, Sun10, Sun1t12, and Sun4t10.

\subsection{Multidimensional (multivariate) data analysis}

Assessment of the interrelations among the selected target variable and individual soil, leaves and meteorological factors was made using several techniques of multidimensional data analysis, namely principal component analysis, cluster analysis, and discriminant analysis (SHARMA, 1996; EVERITT and DUNN, 2001). The statistical technique of correlation analysis was also implemented. The calculations were performed using commercial software packages Statgraphics (ver. 5.1), SPSS (ver. 15), and SAS JMP (ver. 5.1). 


\section{Results and discussion}

\subsection{Correlation analysis}

Table 1 brings a summary of the most important pair correlations, which represents an extract from a much larger correlation table. Each row of the table shows the pair correlation coefficients between the selected target variable (specified in the left column of the table) and the given variable.

Some observations follow from the results given in Table 1. Target variable CROP is mostly influenced by the amount of rainfall and sunshine in September as well as by the nitrogen content in the first phenophase (with a negative correlation!). Target variable SUGAR is mostly influenced by the amount of rainfall and sunshine in April, the temperature level from April to September, the temperature during the whole year and the rainfall in September. SUGAR is also highly correlated with the potassium content in the second phenophase. Target variable ACIDS mostly correlates with the content of calcium and phosphorus in the second and magnesium and potassium content in the first phenophase. The highest and negative correlation is between ACIDS and SUGAR. All mentioned important correlations concern the content of nutrients in vine leaves. The nutrients content in soil (not included into Table 1) seems to be less important for the selected three target variables.

Tab. 1. Most important correlations extracted from the correlation table at the significance level $\alpha=0.05 \%$.

\begin{tabular}{|c|c|c|}
\hline $\begin{array}{l}\text { Target } \\
\text { variable }\end{array}$ & $\begin{array}{l}\text { Highest positive } \\
\text { correlation coefficient } \\
\text { (in parentheses) }\end{array}$ & $\begin{array}{l}\text { Highest negative } \\
\text { correlation coefficient } \\
\text { (in parentheses) }\end{array}$ \\
\hline CROP & Rain9 (0.510) & N1 (0.579), Sun9 (0.500) \\
\hline SUGAR & $\begin{array}{l}\text { Rain4 (0.640), T4 (0.625), } \\
\text { T4t9 (0.620), K2 (0.545), } \\
\text { SumT1t12 (0.545) }\end{array}$ & ACIDS (0.666), Rain9 (0.538) \\
\hline ACIDS & Ca2 (0.563), Mg1 (0.534) & $\begin{array}{l}\text { SUGAR (0.666), K1(0.534), } \\
\text { P2 (0.501) }\end{array}$ \\
\hline $\begin{array}{l}\text { Target } \\
\text { variable }\end{array}$ & \multicolumn{2}{|c|}{ Highest absolute values of the pair correlation coefficient (in parentheses) } \\
\hline CROP & \multicolumn{2}{|c|}{ N1 (0.579), Rain9 (0.510), Sun9 (0.500) } \\
\hline SUGAR & \multicolumn{2}{|c|}{$\begin{array}{l}\text { ACIDS (0.666), Rain4 (0.640), T4 (0.625), SumT4t9 (0.620), } \\
\text { K2 (0.545), SumT1t12 (0.545), Rain9 (0.538) }\end{array}$} \\
\hline ACIDS & \multicolumn{2}{|c|}{ SUGAR (0.666), Ca2 (0.563), K1(0.534), Mg1 (0.534), P2 (0.501) } \\
\hline
\end{tabular}

\subsection{Cluster analysis}

Ward method and squared Euclidean distance were applied since they were found as the most effective way of cluster analysis in preliminary calculations. Obtained 
results of cluster analysis are in good accordance with the performed correlation analysis (Figure 1). Target variable CROP is clustered first of all with the phosphorus content in vine leaves (P1, P2) and the magnesium content in soil (MgMe, MgEuf). Target variable SUGAR is clustered mainly with meteorological variables temperature in April (T4) and in the period April - September (T4t9) and rainfall in April (Rain4). Target variable ACIDS is clustered mainly with the magnesium content in vine leaves (Mg1, Mg2) and the calcium content in soil (CaMe).

\subsection{Principal component analysis}

The sampling sites represented the objects and analytical results and meteorological factors in different year period represented the variables in principal component analysis. They are depicted together in the form of a PCA biplot in Figure 2. It is clearly seen that the objects create three natural clusters, which (according to object numbers in a more detailed analysis) belong to three years of the vine crop: 2000, 2001 and 1999 (from the left to the right).

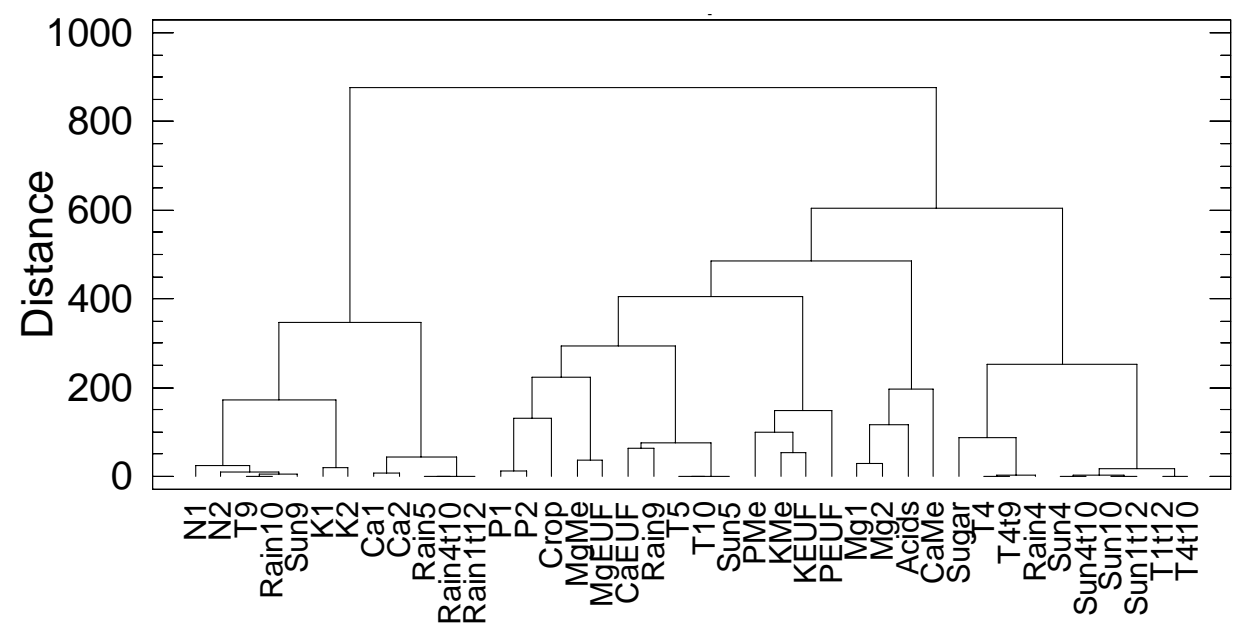

Fig. 1. Dendrogram of cluster analysis of all variables involved in the vine crop and must quality (defined in the text). Ward's clustering technique. Software Statgraphics Plus 5.1.

It was also important to find, which variables are closely related to the three selected target variables, namely CROP (in kg per vine shrub), SUGAR (sugar content in $\mathrm{kg} / \mathrm{hL}$ in must), and ACIDS (content of acids in $\mathrm{g} / \mathrm{L}$ in must), which reflect the crop quantity as well as the must quality.

The smallest angle between CROP and Rain9 indicates the importance of the September rainfall. Almost $180^{\circ}$ angle between CROP and $\mathrm{N} 1$ as well as CROP and Sun9 indicates a large negative dependence of $C R O P$ upon nitrogen content in leaves during the first phenophase as well as amount of sunshine in September. These 
findings are in accordance with the results of correlation analysis, as may be expected. Moreover, the CROP ray points to the 2001 cluster, which means that this year was most successful with regard to the crop of vine.

A close position of SUGAR and Rain4 rays indicates the importance of the April rainfall for the sugar content in must. The importance of the April temperature and the temperature level from April to September is indicated in a similar way. Almost opposite position of the SUGAR and ACIDS rays demonstrates a high content of sugar simultaneously with a low acids content in must and vice versa.

Finally, from the interposition of the samples corresponding to the years 2000 and 1999, respectively, it is visible a large impact of sunshine on the year 2000 samples as well as rainfall on the year 1999 samples.

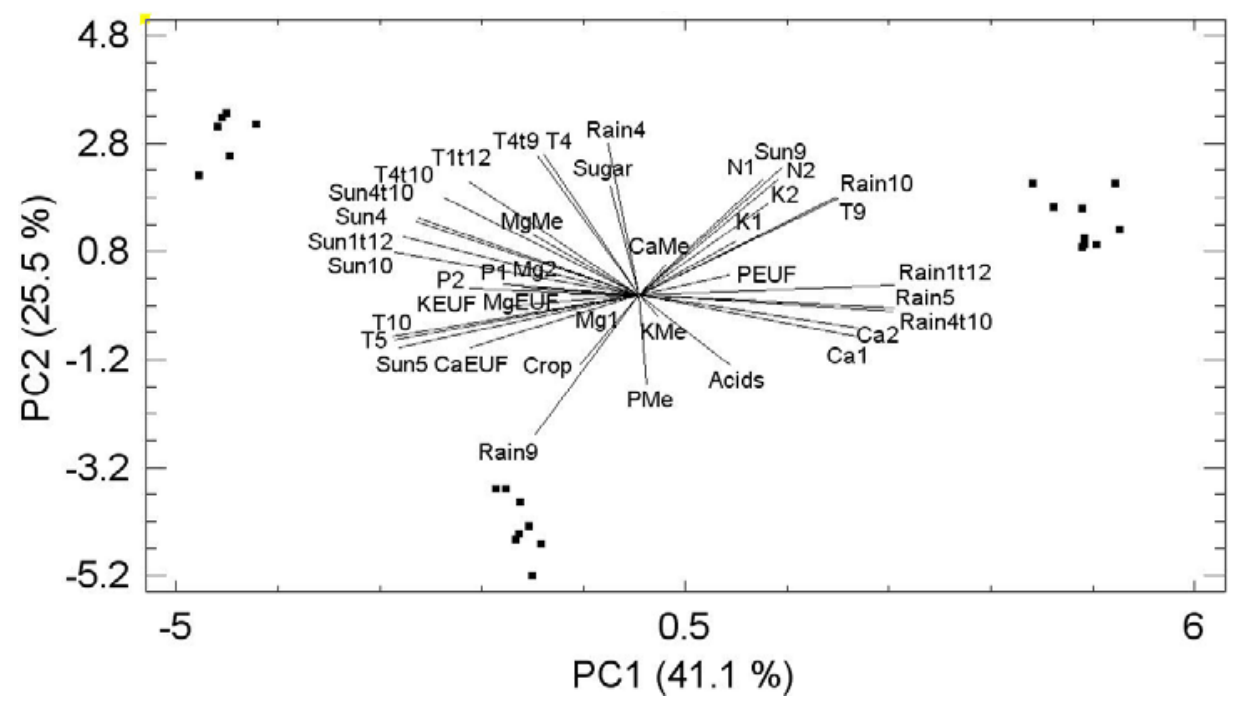

Fig. 2. Biplot PCA showing 40 variables and 24 sampling sites. Three clusters of the sampling sites represent the year of sampling: 2000, 2001 and 1999 - from the left to the right. Software Statgraphics Plus 5.1 .

\subsection{Linear discriminant analysis}

Classification of the objects, which in our case mean the sampling sites, is generally made according to the selected target variable, which has to be categorical. A categorical variable should reflect a known categorization of an object into the chosen category. If it happens, the classification algorithm categorizes the given object into a different category than was the pre-determined category, which is then accompanied by decrease of the classification performance. After calculating the discrimination model, it is possible to use further objects either to validate the performance of the discrimination model by an independent way, or to predict the category where an unknown object should be classified. 
Observed classification performance depended, as expected, on the selected categorical target variable. Figure 3 shows the case when $Y E A R$ was used as the target variable. Here $100.0 \%$ of the originally grouped objects were correctly classified when calculating the discrimination model and $87.5 \%$ of the cross-validated objects were correctly classified using leave-one-out method; altogether 3 objects out of 24 were categorized into a different category then supposed. A $100.0 \%$ performance was observed also in case when CROP was the target variable and all 40 independent variables were used. The same performance was achieved with 20 independent variables without using the meteorological factors. Performance characteristics were obtained also for other combination of variables: soil and meteorological, leaves and meteorological, etc.

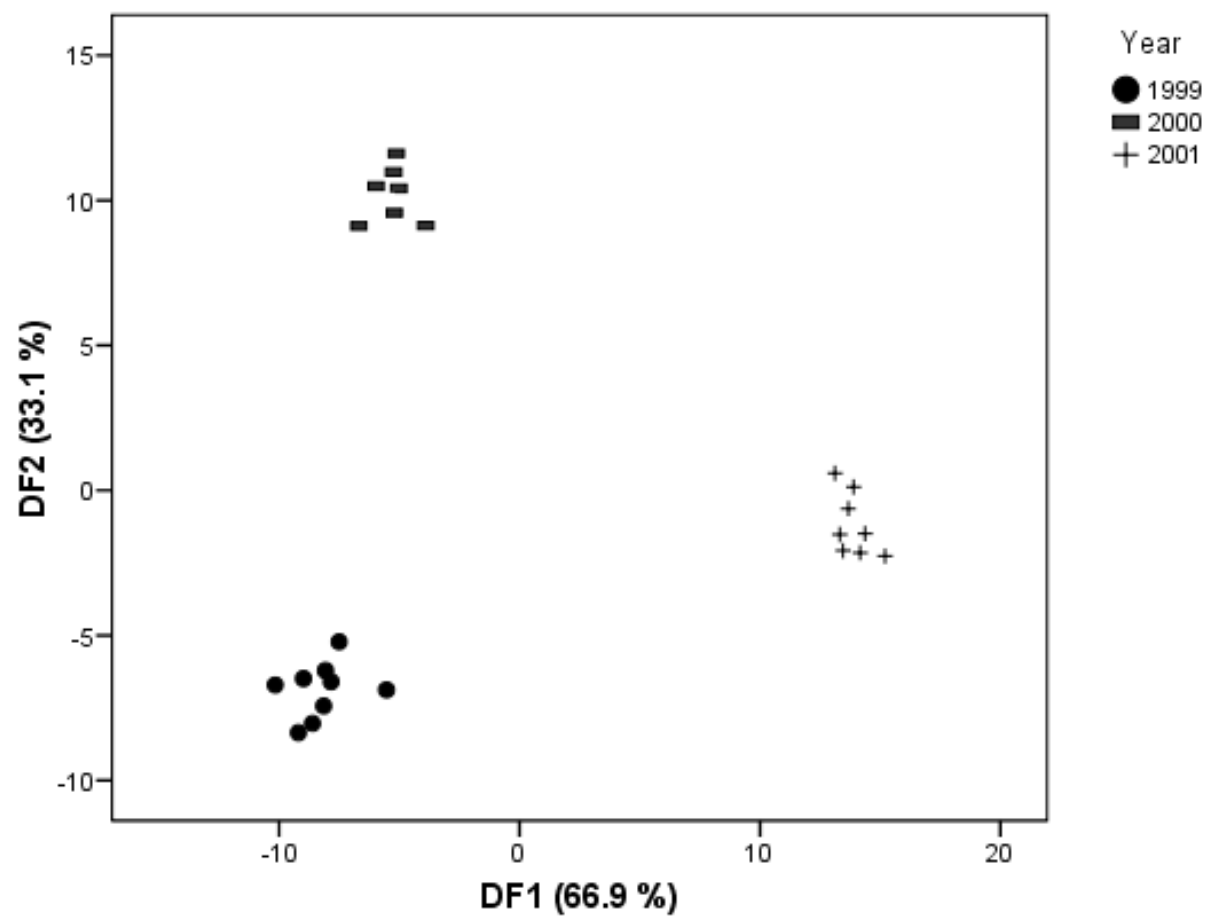

Fig. 3. Plot of discriminant functions (DF's) showing 24 sampling sites clustered by year. A $100 \%$ performance was achieved for the sampling sites classification in discrimination model; $87.5 \%$ performance was achieved for the sampling sites classification using leave-one-out validation. All 40 variables were used in calculations. Software SPSS 15.

\section{Conclusions}

Crop of vine and quality of the produced must are interrelated to the results of elemental analysis of the vineyard soil and the vine leaves. They are also influenced by the meteorological factors like sunshine, rainfall in different months and various years. 
Most important factors influencing the vine crop, the sugar content and the content of acids in must were determined, as well. Sampling sites in South-Western Slovakia were classified according to the target variables CROP, SUGAR and ACIDS by linear discriminant analysis and a very high classification performance was achieved.

Acknowledgment: The support of this work by the grants VEGA 1/3584/06 and AV-4/2025/08 is highly acknowledged.

\section{References}

EVERITT, B.S., DUNN, G.: Applied Multivariate Data Analysis, Arnold, London, 2001.

JONES, J. B.: 1. Laboratory guide for conducting soil tests and plant Analysis, CRC Press, Boca Ranton, 2001.

MELICH M.: Melich-3 soil test extractant: a modification of the Melich-2 extractant. Comm. Soil Sci Plant Anal., 15, 1984, 1409-1416.

MENGEL, K., KIRKBY, E.A.: Principles of plant nutrition, Kluwer Academic Publishers, Bern 2001.

NÉMETH, K.: Recent advances in EUF research (1980-1983). Plant Soil, 83, 1985, 1-19.

NÉMETH, K.: Application of electro-ultrafiltration in agricultural production, Kluwer Academic Publishers, Bern 1982.

NÉMETH, K.: The availability of nutrients in the soil as determined by electroultrafiltration (EUF). Adv. Agronomy, 31, 1979, 155-188.

SHARMA, S.: Applied multivariate techniques, J. Wiley, New York, 1996. 